\title{
La FDIM y los derechos de las mujeres en América Latina: expectativas y alianzas durante la Guerra Fría, 1950-1970
}

\author{
The FDIM and women's rights in Latin America: expectations and alliances \\ during the Cold War, 1950-1970
}

\author{
Yulia Gradskova \\ yulia.gradskova@historia.su.se \\ Universidad de Estocolmo. Facultad de Historia, \\ Suecia
}

Recepción: 30 Noviembre 2019

Aprobación: 06 Mayo 2021

Publicación: 01 Septiembre 2021

Cita sugerida: Gradskova, Y. (2021). La FDIM y los derechos de las mujeres en América Latina: expectativas y alianzas durante la Guerra Fría, 1950-1970. Descentrada, 5(2), e150. https://doi.org/10.24215/25457284e150
Resumen: La Federación Democrática Internacional de Mujeres (FDIM) fue fundada en París, en 1945, con el fin de defender la paz y los derechos de las mujeres y, también, crear mejores condiciones para los niños. Frecuentemente, la Federación fue clasificada como una "organización comunista". Con la ayuda de los documentos de los archivos de Moscú (GARF), el artículo demuestra que la participación de las mujeres latinoamericanas en la FDIM y su influencia dependía de muchos factores, inclusive de la dinámica de la Guerra Fría, de sus orientaciones políticas y, también, no menos importante, de su posición respecto a la Unión Soviética y su política exterior. Pero, de hecho, las activistas latinoamericanas no estuvieron de acuerdo con todas las decisiones de la cúpula pro-soviética y exigían cambios en diferentes aspectos de la organización del trabajo.

Palabras clave: FDIM, Guerra Fría, Movimiento de mujeres, América Latina, URSS.

\begin{abstract}
The Women's International Democratic Federation was founded in Paris in 1945 with the aim of defending peace and rights of women and children. Frequently, theFederation was often addressed as a "communist front". With the help of the documents from the archive in Moscow (GARF) this article shows that the participation of women and organizations from the Latin American countries in the WIDF depended on different factors including the dynamics of the Cold War, their political orientation and their position with respect to the Soviet Union and its foreign policy. But it shows that the female activists from Latin America did not agree with all the political decisions of the pro-Soviet leadership of the WIDF and demanded changes in different aspects of the organization of work in the Federation.
\end{abstract}

Keywords: WIDF, Women's movement, Cold War, Latin America, USSR. 


\section{INTRODUCCIÓN}

La Federación Democrática Internacional de Mujeres (FDIM) fue fundada en París, en 1945, con el objetivo de defender la paz y los derechos de las mujeres, y mejorar las condiciones de vida de los niños. Los estudios anteriores sobre esta organización demuestran que la FDIM fue una organización de mujeres de izquierda muy activa: contribuyó a la declaración de la Convención sobre la Eliminación de Todas las Formas de Discriminación contra la Mujer (Convention on Elimination of All Discrimination Against Women, CEDAW), en 1979, y a la proclamación de 1975 como el Año de la Mujer por parte de la Organización de Naciones Unidas (ONU) (de Haan, 2012; de Haan, 2017; de Haan, 2018; Pieper Mooney, 2013a; Valobra y Yusta, 2017).

Pero, durante los años de la Guerra Fría, la FDIM fue considerada como una organización de la que se servía la Unión Soviética para ejecutar su política exterior expansionista (Kanet, 1988). Como consecuencia de ello, tras el fin de la Guerra Fría,muchas publicaciones dedicadas a la historia de la lucha por los derechos de las mujeres en América Latina no consideraron importante la inclusión de la FDIM (Molyneux y Craske, 2002).

En los últimos años, las historiadoras del movimiento feminista han demostrado que la historia de la FDIM es mucho más compleja que el simplista intento de presentarla como parte del "frente comunista". Aunque tanto la URSS como las mujeres comunistas tenían bastante influencia en la vida interna de la FDIM (Donert, 2014; Valobra y Yusta, 2017; de Haan, 2017), la propia Federación fue una fuerza importante en la lucha contra el fascismo, el racismo y el imperialismo (McGregor, 2016; Pieper Mooney, 2013b; Armstrong, 2016; de Haan, 2017).

Sin embargo, todavía no hay muchas publicaciones que analicen la vida interna de la Federación, incluyendo los conflictos internos y la distribución de poder. Muchas preguntas alrededor de la actividad de la FDIM en el contexto de la Guerra Fría todavía no tienen respuesta. Aún desconocemos, por ejemplo, cómo se tomaban las decisiones dentro de la organización. El nivel de independencia de acciones y decisiones de la FDIM respecto al bloque socialista aún no se ha investigado, como tampoco el margen de maniobra de las organizaciones de los distintos países del "Tercer Mundo" para mantener su propia agenda.

Un artículo no puede responder a todas estas preguntas, pero mi objetivo aquí es contribuir a la búsqueda de respuestas en lo referente a la participación de las mujeres latinoamericanas a través de la documentación contenida en el Archivo Estatal de la Federación Rusa en Moscú (Gosudarstvennyi Arkhiv Rossiiskoi Federazii, GARF) ${ }^{1}$. Todo este material sobre las actividades de la FDIM, en general, y su trabajo en América Latina, en particular, todavía no ha sido analizado.

Para mi análisis, uso el enfoque teórico que no contempla la Guerra Fría como la mera confrontación de dos bloques principales, sino que considera fundamental prestar atención a las dimensiones globales, culturales y sociales de este fenómeno (Westad, 2005; Hajimu, 2015; Autio-Sarasmo \& Miklossy, 2011). Me propongo examinar, de este modo, la lucha por los derechos de las mujeres que llevaba a cabo la FDIM en el contexto de la Guerra Fría en el "mundo entero". También voy a examinar cómo esta idea fue transformada y adoptada durante el período de 1950-1970 en relación con América Latina.

Muchos historiadores e historiadoras tuvieron interés en la FDIM en lo referido a sus actividades para apoyar los países no-europeos, como Vietnam o Indonesia (McGregor, 2013 y 2016) o muchos países de América Latina (Valobra \& Yusta, 2017). Su investigación se basó, en gran parte, en los archivos locales de sus países respectivos. A diferencia de estos estudios, mi artículo está basado principalmente en los documentos en ruso del Archivo Estatal de la Federación Rusa (GARF). La organización de las mujeres soviéticas Comité de las Mujeres Soviéticas, miembro fundador de la FDIM - tenía una representante permanente en su Secretaría (en la sede principal de la FDIM, primero en París y, después de 1951, en Berlín). Además, el ruso fue uno de los idiomas oficiales de la organización. Dado que los archivos centrales de la FDIM, por ahora, no están accesibles, ${ }^{2}$ el archivo del Comité de las Mujeres Soviéticas en Moscú puede ser una fuente 
de información muy importante porque tiene muchos volúmenes de los protocolos de los órganos dirigentes de la FDIM traducidos al ruso y, también, una cantidad importante de correspondencia dentro de la oficina principal en Moscú y la representante soviética en la Secretaría de FDIM fuera del país (Gradskova, 2019).

Entre estos documentos, hay muchos que de una u otra manera están discutiendo actividades y relaciones de la FDIM con las organizaciones de mujeres en los países de América Latina. También, los documentos del archivo de Moscú pueden ayudarnos a entender mejor el papel de la Unión Soviética en esta organización transnacional, especialmente con la ayuda del análisis de correspondencia entre representantes soviéticas. Esta correspondencia, en parte, está clasificada y contiene muchos comentarios de carácter privado alrededor de los conflictos internos en la cúpula de organización. A veces, los documentos preservados parecen ser demasiado subjetivos y particulares, pero la lectura atenta puede ayudar a contextualizar acontecimientos y personalidades políticas en su ambiente emocional y social.

Al mismo tiempo, los documentos preservados en Moscú ayudan a entender cómo las mujeres de América Latina participaban en las decisiones centrales de la Federación. Finalmente, los documentos de archivos de Moscú pueden contribuir al conocimiento más profundo sobre el lugar que los problemas y las demandas de las mujeres latinoamericanas tenían en el trabajo diario de la organización. La mayoría de estos documentos todavía no están analizados y son los que forman la base principal para mi investigación.

Junto con la documentación de los archivos, analizo la publicación oficial de la Federación: la revista Women of the Whole World (WWW en inglés) / Zhenshchiny mira (ZM en ruso). Además de las ediciones en inglés y en ruso, esta revista se traducía al español, el francés y el alemán; no obstante, hasta la fecha sólo he encontrado ejemplares en ruso o inglés. La revista empezó a publicarse en 1951, pero tuve acceso a algunos números de 1953 y a colecciones anuales más completas a partir de 1959. El diseño y el número de revistas publicadas cada año durante el período analizado variaban de doce, en 1958 — periodicidad mensual一, a seis durante los años 1960 y 1970.

En la línea de Ann Stoler (2009), quien en su estudio de los archivos coloniales en los Países Bajos demuestra cómo es posible usarlos para analizar la construcción del conocimiento sobre "el otro" en el sistema colonial, planteo que el uso del archivo de la organización de mujeres soviéticas de la FDIM y la revista de la Federación pueden ayudar a entender y deconstruir la forma en que se representan los "derechos de las mujeres" y "las mujeres de América Latina” en el lenguaje oficial de la Federación. También uso los materiales de dicho archivo para estudiar las diferencias en las posiciones y acciones de las participantes de la Federación de América Latina, por un lado, y de las representantes soviéticas y europeas, por el otro.

Todo el período analizado discurre en el contexto de la Guerra Fría; por consiguiente, la actividad en favor de las mujeres de la Federación transnacional quedó marcada por este contexto, a la vez que influyó el desarrollo y la conflictividad política de la Guerra Fría.

Los trabajos de Valobra \& Yusta (2017), Valobra (2014), de Haan (2017), Pieper Mooney (2013b) demuestran que las mujeres de América Latina eran activas en la FDIM y que algunas representantes de América Latina eran elegidas como vice-presidentas y secretarias generales de la organización (de Haan, 2017, Pieper Mooney, 2013b). Más específicamente, de Haan demuestra que, aunque las organizaciones de mujeres de algunos países latinoamericanos eran miembros de la FDIM desde la fecha de su fundación (de Haan, 2017), su participación masiva se realiza desde la mitad de los años 1950. En 1959, tuvo lugar en Santiago de Chile el primer congreso de mujeres latinoamericanas apoyado por la FDIM (Valobra, 2014) y, durante el periodo posterior, la FDIM regularmente organizó encuentros latinoamericanos. A finales de la década de 1970, una nueva institución apoyada por la FDIM fue fundada en América Latina: el Centro Regional de la FDIM para América y El Caribe, que empezó a funcionar en la Habana, Cuba, en 1978. Esto significaba el crecimiento importante del rol de Cuba como el líder regional de la FDIM. Finalmente, se puede suponer que el inicio del proceso de reformas en la Unión Soviética (Perestroika), en 1985, contribuyó al papel creciente de Cuba en el continente: después de la caída del muro de Berlín (1989) y la descomposición de la Unión Soviética (1991), la sede de la FDIM en Berlín fue cerrada, su revista oficial dejó de publicarse y la cantidad 
de los miembros de la organización se redujo drásticamente. ${ }^{3}$ Pero, de acuerdo con la página web cubana, la Federación de Mujeres Cubanas (FMC) sigue siendo miembro activo de la FDIM cuya sede actual está situada en El Salvador. ${ }^{4}$ Entonces, a continuación, espero precisar también este crecimiento del papel de América Latina en la organización fundada en Europa en 1945.

Comenzaré por el análisis de las representaciones de los problemas de mujeres de América Latina y las actividades de las organizaciones femeninas en esta región basándome en la revista oficial de la FDIM. Luego, analizaré cómo cambió el carácter de la participación de las mujeres latinoamericanas durante el periodo de investigación. ¿Qué países y problemas reciben más atención en la prensa de la FDIM? ¿Cómo eran representadas las mujeres de América Latina en los órganos dirigentes de la federación y qué transformaciones se pueden observar? ¿Cómo cambia la importancia de algunos países para la Federación?

\section{LAS MUJERES LATINOAMERICANAS EN LA REVISTA DE LA FDIM EN LOS AÑOS 1950-1960}

Comenzaré con una visión general sobre cómo la revista representaba los problemas de las mujeres latinoamericanas y su activismo. No tuve acceso a todos los números, pero mi interés principal no radica en la cantidad de materiales sobre las mujeres de América Latina en sí, sino en los problemas abordados y las soluciones que se proponían. ${ }^{5}$ También, he centrado mi atención en las actividades emprendidas y en las activistas que eran visibles.

En el año 1953, la revista de la FDIM publica materiales informativos cortos bastante uniformes, cuyo contenido se centra en las acciones y celebraciones internacionales. Busca demostrar que las mujeres latinoamericanas están participando también, junto con mujeres de otros continentes, en las actividades transnacionales. De este modo, las representantes latinoamericanas eran presentadas, ante todo, como las defensoras de la paz. Los países latinoamericanos mencionados con mayor frecuencia en la revista son Chile, Cuba, Brasil y Argentina. En el número 1 de 1953, se puede leer, por ejemplo, que las chilenas están en contra del nuevo pacto militar que su país quiere firmar y que hay muchos niños pobres (así lo declara la representante chilena en el consejo de la FDIM, s.n., ZM, 1953, 1, p. 8). En el mismo número, se publicó un largo artículo dedicado al Congreso de Partidarias de la Paz en Viena, apoyado por la FDIM. En parte de este artículo, la brasileña Elisa Branco relata cómo ha sido arrestada por su agitación contra la decisión del gobierno de Brasil de mandar tropas a Corea (ZM, 1953, 1, p. 14). Otro artículo sobre Branco nos informa que ha recibido el premio Stalin y se presenta su biografía (ZM, 1953, 1, p. 17). ${ }^{6}$ En el mismo número, también se puede leer que 14 mujeres de la Federación Democrática de Mujeres Cubanas habían participado en el consejo de la FDIM en Viena (ZM, 1953, 1, p. 25). El número 3 de 1953, no tiene materiales sobre América Latina, a excepción de una carta muy corta sobre cómo las mujeres de Brasil están en contra de la guerra (ZM, 1953, 3, p. 21).

Se puede observar que, en la mayoría de los artículos publicados, las mujeres latinoamericanas presentadas por la revista pueden ser calificadas como marcadas por una posición ideológica bien definida. En el contexto de las divisiones de la Guerra Fría, esta posición era claramente a favor del campo del socialismo estatal y la Unión Soviética. Entonces, se puede decir que estos artículos tenían una función muy importante para la Federación: demostraban que las mujeres de América Latina estaban participando en una lucha común con las mujeres progresistas y democráticas de todo el mundo. Pero los materiales publicados en la revista no comentaban mucho alrededor de otras organizaciones en estos países que luchaban por los derechos de mujeres ni alrededor del número de activistas que apoyaban a la FDIM en cada país. Parece que la posibilidad de contar con el apoyo de algunas mujeres en el continente, a pesar de estar situado tan lejos de la URSS como América Latina, fue importante para una organización como la FDIM.

Los informes sobre los países latinoamericanos no solamente aumentaron en la revista de la FDIM en los años posteriores, sino que cambiaron de estilo y modo de presentación. Cabe vincular esto tanto con 
los cambios en la Unión Soviética tras la muerte de Stalin y el consiguiente proceso de "desestalinización", que contribuyó a los conflictos dentro de los partidos comunistas en el mundo, como con el crecimiento del interés de las mujeres europeas hacia países fuera de Europa, dentro del contexto de emergencia de los movimientos anticoloniales. Por ejemplo, un artículo publicado en 1958 describe la situación en Cuba y afirma que la dependencia del país del monocultivo del azúcar, al cual se ve obligado por el imperialismo mundial, contribuye a la pobreza de su población (ZM, 1958, 6, p. 25). Mientras que el número 7-8, sólo menciona brevemente que la delegada de Ecuador participó en el congreso de la FDIM en Viena (ZM, 1958, 7-8, p. 9), el número 9 del mismo año publicó un artículo dedicado a una escritora salvadoreña, Lilian Jiménez. La nota también mostraba los problemas cotidianos que sufrían las mujeres de este país, incluyendo la falta de seguro social y de asistencia médica, así como la persecución policial a la que se veían sometidas las vendedoras ambulantes (ZM, 1958, 6, p. 9).

Un artículo bastante largo, publicado en el número 10 del mismo año, tenía el objetivo de demostrar la participación de las mujeres latinoamericanas en la FDIM. A muchas de ellas le ofreció la oportunidad de cooperar con otras mujeres de diferentes partes del mundo. La participación en la FDIM les prometía amplios contactos con mujeres de todos los continentes. El artículo de Lidia da Cunha, de Brasil, cuenta su viaje a China (ZM, 1958, 10, p. 13). Según el texto, este viaje le impresionó mucho y pudo observar "la unión completa del pueblo y el Gobierno".

Las publicaciones sobre la situación de mujeres y niños en los países latinoamericanos presentan, desde entonces, una mayor tendencia a explorar de forma más profunda las problemáticas específicas de diferentes países. Estos artículos aumentaron especialmente en 1959, año de la Revolución cubana. En el número 3 de la revista, se publicó una nota de la profesora María Luisa Vincentini sobre la revolución, donde se explicaba cuán importante había sido siempre la independencia para los cubanos y cómo ahora los libertadores en armas habían tomado el poder (ZM, 1959, 3, pp. 20-21). Las fotografías que acompañaban al texto mostraban a las cubanas incluso con uniforme militar. El mismo año, la revista publicó también varios artículos sobre algunos otros países latinoamericanos, incluyendo fotorreportajes sobre la difícil vida de las campesinas en Brasil (ZM, 1959,7,pp. 17-18), así como un artículo sobre la historia del desarrollo del movimiento feminista en Argentina (ZM, 1959, 7, pp. 30-31). En los primeros números de 1960, la revista publicó un artículo sobre Ana Betancourt, la heroína de la Guerra de Independencia cubana (ZM, 1960,1, p. 39), y otro escrito por Izula sobre la situación de las mujeres en Brasil (ZM, 1960, 2, pp. 22-25). En 1961, apareció un artículo sobre Guatemala (ZM, 1961, 2, pp. 22-23) y un texto informativo sobre la situación de las mujeres en Paraguay (ZM, 1961, 3, pp. 9-10). A partir de ese año, la mayoría de publicaciones también están firmadas por autoras provenientes del continente americano.

Pero parece que, desde 1959, las mujeres y las dirigentes cubanas obtuvieron un lugar especial en la revista. Las lectoras podían obtener información sobre diferentes aspectos de la vida de las mujeres en Cuba y sobre sus dirigentes. Por ejemplo, el número 2 de 1961 incluye un artículo de Luis Cabrera sobre la patriota cubana Ángela González, participante de la lucha militar contra la dictadura (ZM, 1961, 2, pp. 20-21); el artículo de Onelia Aguilar dedicado a Vilma Espín, la dirigente de la nueva organización de mujeres cubanas posrevolucionaria constituida en 1960 (ZM, 1963, 1, pp. 6-7); y también, el artículo del corresponsal europeo de la revista sobre La Habana y las reformas cubanas (ZM, 1963, 3, pp. 32-33). El texto sobre Espín empieza relatando el Congreso de Mujeres de América Latina que tuvo lugar en La Habana, en enero de 1963. La autora quería demostrar el lugar especial de Cuba en el movimiento femenino en el continente. A continuación del artículo, las lectoras podían informarse sobre la vida de Vilma Espín, presentada como una mujer política extraordinaria. No obstante, el texto de la revista de la FDIM la mostró no sólo como una dirigente heroica, sino también como una mujer común y corriente. Parece que el papel de Espín como madre -la nota cuenta que tiene una hija pequeña- fue no menos importante para las redactoras de la revista que su papel como combatiente y dirigente revolucionaria. Esta presentación correspondía y apoyaba la ideología maternalista de la FDIM de acuerdo a la cual la maternidad fue una función natural para las mujeres. Esta 
función hacía que las mujeres pudieran actuar en la política actualizando valores diferentes de los masculinos (Pieper-Money, 2013b).

En los años 1960, la FDIM expandió el alcance del número de organizaciones y países participantes en sus actividades. Esta fue una de las consecuencias del proceso de descolonización en África y Asia. En 1967, la Federación pudo obtener el estatus especial de organización no-gubernamental en la ONU.7 También, fue en esos mismos años que representantes de organizaciones de mujeres en América Latina tomaron posiciones importantes en la dirigencia de la Federación, como se verá en el apartado 4. En este período, algunas mujeres destacadas -como Fanny Edelman, de nacionalidad argentina, que se transformó en secretaria general de la FDIM en los años 1972-1978 (Pieper Mooney, 2013; Edelman, 1996) - ocupan un lugar importante en las publicaciones de la revista. También, la revista de la FDIM publica reportes analíticos sobre la situación de las mujeres en el continente y en el mundo, escritos por dirigentes y activistas latinoamericanas. Por ejemplo, el número 1 de 1969 fue dedicado a las mujeres de América Latina. El número dedicado al Congreso de la FDIM (Helsinki, 1969) publicó la ponencia de Martha López Portillo de Tamayo, representante de la Unión Nacional de Mujeres Mexicanas (WWW, 1969, 3, pp.38-43). En este artículo, López Portillo anuncia una nueva etapa de la lucha por la igualdad de las mujeres en el mundo: todas las "sociedades modernas" han aceptado la igualdad legal de los ciudadanos independientemente de su raza, nacionalidad o sexo; la igualdad social y económica de las mujeres en la vida real puede ser realizada solamente como resultado de la "transformación profunda de la sociedad entera". López Portillo insistió en que todas las mujeres tenían que participar activamente en el "desarrollo de la sociedad", pero notó que las costumbres tradicionales frecuentemente les impedían hacerlo (WWW, 1969, 3, p. 39). La ponencia también defendía la importancia de participación de las mujeres en la política y analizaba los problemas que las mujeres encontraban en esta esfera. En realidad, de acuerdo con López Portillo, había 114 países donde ellas tenían el derecho de participar en las elecciones, pero el número de las mujeres que tenían cargos políticos importantes seguía siguiendo bajo (WWW 1969, 3, p. 41). Acusando al colonialismo por la herencia de subdesarrollo en África, López Portillo también indicaba varios factores negativos que agravaban la situación de muchas mujeres, incluida la poligamia y la prostitución. Estos factores estaban amenazando la dignidad y la salud de la mujer (WWW, 1969 3, p. 39). Así, se puede decir que esta ponencia demostraba que algunas activistas latinoamericanas se convertían en portavoces de las ideas de la Federación, un desarrollo importante comparando con los artículos cortos publicados por la revista oficial de la FDIM a principios de los años 1950.

Es muy interesante notar que esta ponencia también contenía cierta crítica a los países del "campo socialista", algo extraño en esta revista que siempre publicaba materiales sobre la Unión Soviética como un ejemplo extremadamente positivo de la solución de todos los problemas de las mujeres. En este artículo, se puede leer: "even in the most advanced socialist countries today, the woman devotes $60 \%$ more time to domestic labours than the man does" (WWW, 1969, 3, p. 40).

En la base de esta breve revisión alrededor de los cambios de la representación de América Latina en la revista oficial de la FDIM, se puede decir que su rol evolucionó de servir como un ejemplo ilustrativo de participante en actividades comunes de "mujeres del mundo entero", hacia un retrato más diversificado y concreto. Algunas organizaciones y líderes femeninas de la región empezaron a ser presentadas por la revista como muy competentes y ejemplos para otros países y regiones. En el siguiente apartado, me concentraré en los documentos del archivo de Moscú para investigar cómo se realizaba en la práctica la participación de las latinoamericanas en los órganos dirigentes de la FDIM y qué conflictos y problemas generaba.

\section{LA VIDA INTERNA DE LA FDIM Y LA PARTICIPACIÓN DE ACTIVISTAS LATINOAMERICANAS}

El archivo de Moscú conserva muchos protocolos de las reuniones de los órganos dirigentes de la FDIM que actuaban en los períodos entre los congresos: el Consejo que incluía un representante de cada país miembro de la organización, y la Mesa, sección de representantes electas. Ambos órganos dirigentes se 
reunían para discutir los problemas más importantes del trabajo de la Federación. En los años 1950, después de que la sede principal de la Federación tuviera que trasladarse a Berlín, los encuentros habitualmente tenían lugar en uno de los países "socialistas". En las reuniones de los órganos directivos de la FDIM, las discusiones más intensas se corresponden con el período desde finales de los años 1950 a principios de los años 1960.

Este fue un tiempo de cambios rápidos, principalmente en lo que atañe al sistema colonial (más de cuarenta países en África y Asia consiguieron su independencia en estos años). Como resultado de la descolonización, muchas organizaciones femeninas nuevas ingresaron en la FDIM durante ese período. Esto significaba que la agenda programática introduciría temas nuevos: problemas de las luchas por la independencia, el racismo o las campañas contra el analfabetismo se discutieron en los documentos oficiales de la FDIM. Por ejemplo, en septiembre de 1962, en Tashkent (Uzbekistán, que formaba parte de la URSS en aquellos años), tuvo lugar un seminario para la educación de las mujeres de África (GARF, 3 776, p. 27). En 1970, Women of the Wholw World, publicó un número especial dedicado a la conferencia sobre analfabetismo en Jartum, Sudán (WWW, 1970, p. 3). Por otra parte, este fue un período de cambios profundos en la Unión Soviética y en el "campo socialista". La muerte de Stalin (1953), la crítica del estalinismo en el congreso del Partido Comunista soviético (1956), la intervención militar soviética en las sublevaciones o revoluciones de Hungría y Checoslovaquia (1956 y 1968), así como la construcción del Muro de Berlín en 1961, provocaron muchas discusiones alrededor de los fundamentos ideológicos de la Federación (Valobra, 2014).

Los documentos del archivo de Moscú contienen aún más evidencia sobre las limitaciones para la actividad de la Federación en el contexto de las confrontaciones ideológicas de la Guerra Fría. Por ejemplo, la correspondencia entre la representante soviética en la sede de la FDIM en Berlín, Zoia Ivanova, y Nina Popova, ${ }^{8}$ la dirigente del Comité de Mujeres Soviéticas en Moscú, demuestra que las semanas posteriores a la presentación de Jrushchov en el XX Congreso del Partido Comunista Soviético (Moscú, 1956) fueron muy duras para las representantes del Comité de las Mujeres Soviéticas en el Secretariado: la ausencia de instrucciones claras de Moscú y el carácter secreto de la ponencia contribuyeron a la situación cuando las representantes soviéticas no pudieron responder a múltiples preguntas de colaboradoras del Secretariado de otros países. Zoia Ivanova describe así la situación:

Le quiero informar que nuestra situación en el Secretariado se hizo más difícil: nuestras amigas han escuchado mucha información nueva por radio y televisión y nos preguntan sobre el culto a la personalidad, y especialmente, sobre el papel de Stalin en la historia de nuestro partido y nuestro país. También, hacen preguntas sobre el rol de Stalin en la historia del movimiento obrero. [...] Todo esto provocó muchas discusiones de todo tipo (GARF 4 106, p. 64; 24.03.1956).

La correspondencia con Moscú demuestra, también, que algunas mujeres de los países europeos expresaron la necesidad de cambiar las reglas de la Federación, inclusive, querían hacer el programa de la FDIM más abierto a diferentes tipos de organizaciones de mujeres y exigían más democracia interna (Gradskova, 2019). En esta situación, el papel de las mujeres de los países extra-europeos creció significativamente. Muchas organizaciones en África, Asia y América Latina tenían que actuar en las condiciones de la guerra anticolonial o en la clandestinidad, no tenían recursos económicos para el mantenimiento de la organización y estaban muy interesadas en contactos internacionales y en la solidaridad de mujeres de otros países. Además, es importante notar que, en muchos casos, a las organizaciones de los países fuera de Europa les faltaban conocimientos sobre los problemas europeos y sobre la historia del estalinismo soviético, tampoco era envueltos en la discusión sobre el futuro de partidos comunistas. Para las representantes de estos países, la FDIM era una organización con un programa atractivo que ponía en agenda la igualdad de las mujeres y la protección social de la maternidad. Además, a través de la participación en las reuniones, las representantes de organizaciones de mujeres de África, Asia y Latinoamérica podían obtener los conocimientos necesarios y, también, contar con el apoyo y solidaridad de una organización poderosa y transnacional.

La participación masiva de las mujeres de los países que declararon su independencia en los años 1950-1960 provocó una discusión amplia acerca de la relevancia de los conceptos ideológicos de la FDIM. Algunas 
representantes de los países latinoamericanos participaban muy activamente en estas discusiones. Por ejemplo, uno de los aspectos importantes era el uso del lenguaje ideológico de los documentos oficiales de la FDIM y los problemas con la capacitación de las mujeres. Muchas mujeres en diferentes países latinoamericanos podían estar interesadas en más posibilidades y derechos, pero estaban asustadas de los términos ideológicos de los documentos oficiales como "el imperialismo". ${ }^{9}$ En el contexto de la propaganda oficial de sus países, el uso de ciertos términos ideológicos calificaba la actividad de organizaciones femeninas como organizaciones antigubernamentales. En su país de origen esto podía perjudicar sus organizaciones o, incluso, exponerlas a la represión. Como los documentos demuestran, algunas delegaciones latinoamericanas, como la chilena, apoyada por representantes de muchos países africanos y asiáticos, insistió en un programa más inclusivo de la FDIM y menos influido por la confrontación de la Guerra Fría. Durante la reunión de la Mesa de la FDIM en Moscú, en 1963, la representante de Chile dijo:

\begin{abstract}
Nuestra organización quiere ser una organización para las masas de mujeres. Al principio, nosotras también hemos hablado sobre el imperialismo. Pero entendemos que las mujeres chilenas son menos avanzadas que las mujeres de Venezuela y de Cuba y por esto no podemos hablar con ellas el idioma de las mujeres comunistas. La mayoría de ellas no puede comprender que significa 'la lucha contra el imperialismo'. Entonces, nosotras hablamos con estas mujeres sobre los problemas que tienen, nosotras hemos decidido usar esta táctica. Tenemos que usar el lenguaje que puede atraer a las mujeres de otras organizaciones, así la FDIM puede crecer. Cuando estas mujeres estén con nosotras, vamos a hacer el cambio cualitativo (GARF, 3, 1027, p. 20).
\end{abstract}

Las delegadas latinoamericanas, junto con las de países independientes de África y Asia, insistían en que las mujeres de los países no-europeos tenían que tener más lugar en los órganos dirigentes de la Federación. A finales de los años 1950, algunas representantes de los países latinoamericanos se pronunciaron por una participación más activa. Por ejemplo, los protocolos de la reunión de la Mesa de FDIM en Praga, en octubre de 1959, contienen la intervención de Izula Gerhard, de Brasil. En su discurso, Gerhard insistía en que era importante cambiar la formación de la dirigencia de la FDIM:

No es correcto que solamente dos representantes de América Latina sean miembros de la dirigencia. Esto previene que la cúpula de la FDIM pueda entender plenamente los problemas que tienen las mujeres de uno u otro país. El Secretariado tiene que prestar atención especial a América Latina y corregir los errores anteriores (GARF 3221, p. 8).

La misma idea - de acuerdo con la comunicación secreta entre la representante de la URSS en el Secretariado de Berlín y el Comité de las Mujeres Soviéticas - fue informada a la cúpula de la FDIM a través de Adela Betinelli, quien participaba en nombre de la delegación del Partido Comunista argentino en las celebraciones del décimo aniversario de la Revolución china en Pekín, en 1959. De acuerdo a este mensaje:

El papel principal en el movimiento democrático mundial lo tienen las luchas de los pueblos de Asia, África y América Latina, y no la lucha de los pueblos europeos. Esto ha de ser visible en la composición de las estructuras dirigentes [de la FDIM]. [...] Las representantes europeas no pueden representar plenamente las demandas de los pueblos de estos países (GARF, 4 134, p. 136).

A continuación, la carta observa también que, según "los amigos argentinos", el discurso de VaillantCouturier, que representaba a la FDIM en Pekín, fue el discurso de "una mujer francesa” y no el que hubiera correspondido a la vicepresidenta de la FDIM (GARF, 4 134, p. 136).

Como he notado antes, la Revolución Cubana (1959) tenía un papel especial en el desarrollo del movimiento de las mujeres en el continente latinoamericano y para la FDIM. Valobra señaló que "las barbudas" -encabezadas por Vilma Espín - fueron el centro del Congreso Latinoamericano de Mujeres que tuvo lugar en Santiago de Chile, en noviembre 1959 (Valobra, 2014, p.9). Los documentos de archivos hacen suponer que las mujeres cubanas obtuvieron un lugar importante en la Federación de manera paulatina y que este proceso se desarrolló paralelamente con el acercamiento ideológico entre la Unión Soviética y Cuba. El documento que describe la historia de las relaciones de la FDIM y del Comité de las mujeres soviéticas con la nueva organización de mujeres cubanas, creada en 1969 (Chase, 2015), muestra que, al principio, los 
contactos tenían un carácter limitado a ciertos temas. Por ejemplo, las mujeres cubanas estaban interesadas en el desarrollo de círculos infantiles y en las condiciones de trabajo de las mujeres en la URSS (GARF 3 5799, pp.7-8). Pero desde 1970, las relaciones dentro de las organizaciones femeninas de la URSS y de Cuba empiezan a ser más estrechas, la colaboración se hizo más amplia y el documento escribe más sobre el interés de las cubanas en "la solución del problema de las mujeres" en la URSS (GARF 3 5799, p. 6). Las relaciones más estrechas con la organización de las mujeres soviéticas parecen coincidir en el tiempo con el papel más importante que Cuba empezó a tener en la Federación: el año 1973, la primera Mesa de las dirigentes de la FDIM tuvo lugar en América Latina, en la Habana (Protocolos de la Mesa de la FDIM, GARF 33162$)^{10}$. Cerca de veinte dirigentes de la organización encabezadas por la presidenta de la FDIM, Hertta Kuusinen, eran recibidas por la Federación de las Mujeres Cubanas en presencia de Vilma Espín y Fidel Castro. En su ponencia de apertura de la reunión, Espín dijo:

Por primera vez, se celebra la Mesa de la FDIM en un país de América Latina y ello tiene, sin lugar a dudas, una significación especial ya que refleja la importancia de nuestra organización internacional en la lucha de las mujeres en este continente por un futuro mejor para sus pueblos (GARF 3 3162, p. 9).

Hertta Kuusinen también notó en su ponencia que esta era la primera vez en la historia de la FDIM que la reunión de la Mesa tenía lugar en América Latina (GARF 33162, p. 34). Parece haber sido un acontecimiento bastante grande para una Federación que quería representar a las mujeres "del mundo entero", pero tenía la mayoría de su actividad dirigente en Europa Occidental.

En la Habana, las dirigentes de la FDIM discutían los problemas corrientes, incluyendo la distribución de la revista "Women of the Whole World” en los países de África y la preparación de la organización para el año de la mujer declarado por ONU para 1975. La ponencia sobre el último tema fue realizada por Fanny Edelman, representante de Argentina (GARF 3 3162, p. 13-20). A propósito de ella, voy a discutir los problemas de poder interno de la Federación y el papel de mujeres de América Latina como dirigentes de la FDIM en el siguiente apartado del artículo.

\section{LATINOAMERICANAS EN LA DIRIGENCIA DE LA FDIM y LAS DINÁMICAS DE PODER}

Sabemos que las mujeres de varios países latinoamericanos, en diferentes periodos, participaron en la dirigencia de la FDIM. ${ }^{11}$ Aquí voy a usar los documentos del archivo para comentar sobre la nominación de algunos casos y de su influencia interna. Sin embargo, discutiendo la dinámica de poder en la Federación, es importante tomar en cuenta no solamente cualidades y competencias personales, sino también, el contexto de la Guerra Fría que -en muchos casos- significaba que las posiciones ideológicas y los cargos en los partidos políticos tenían la misma o mayor importancia que las calificaciones personales.

Los documentos del archivo de Moscú demuestran que las dirigentes latinoamericanas como otras de la cúpula de la FDIM que eran miembros del partido comunista, frecuentemente, tenían una responsabilidad doble: eran obligadas a seguir las decisiones del partido y, al mismo tiempo, a pensar y actuar como las dirigentes de la organización transnacional de mujeres. En algunos casos, eran los partidos comunistas de uno u otro país los que decidían quién iba a representarlos en la FDIM. Por ejemplo, de acuerdo con la carta enviada a Moscú, así fue en el caso de Olga Luzardo, una comunista venezolana que, en 1963, tenía que empezar a trabajar en el Secretariado en Berlín. La representante soviética en el Secretariado en Berlín, Zoia Lebedeva, comunicó en su carta que ella había tenido una conversación privada con el Secretario General del Partido Comunista de Venezuela, camarada Faría, durante su visita a Berlín el 22 de enero. Faría comunicó a Lebedeva que Olga Luzardo "es una persona competente y con mucha experiencia, tiene buenas tradiciones de luchar [ella fue encarcelada como presa política durante un tiempo]". El secretario del Partido Comunista venezolano también comunicó que Luzardo era miembro del Comité Central, diputada del parlamento y tenía educación universitaria. Es interesante notar que el factor de conocimiento del idioma ruso parecía 
también importante: de acuerdo a Faría, Luzardo estudiaba en Moscú y hablaba ruso (GARF 79284169 , 1963). Entonces, se puede entender que, en el caso de algunas representantes latinoamericanas, como el de Luzardo, el trabajo en la organización transnacional de mujeres constituía una parte del trabajo político.

A pesar de que los puestos y las posiciones ideológicas tenían mucha influencia para las posibilidades de ser dirigentes de la FDIM, la correspondencia dentro de las representantes soviéticas en el Secretariado de la FDIM en Berlín y Moscú demuestra también que la distribución del poder entre Moscú y las organizaciones de mujeres latinoamericanas (y de otros países) no era totalmente predefinido por Moscú, aunque se negociaba constantemente. Esto pasaba, inclusive, en casos en los que las organizaciones de mujeres en países latinoamericanos tenían dirigencia de mujeres comunistas. Por ejemplo, un documento de 1961 marcado como "documento secreto", describe a una representante argentina en el Secretariado, Adela Betinelli. De acuerdo con este reporte soviético, Betinelli, integrante del Comité Central del Partido Comunista de Argentina y de la Unión de Mujeres Argentinas (UMA), fue responsable del trabajo del Secretariado en los países latinoamericanos. Pero su actividad recibió muchas críticas en este documento soviético, ya que fue pasiva respecto de todas las discusiones sobre los problemas de las mujeres excepto de las que se refieren a América Latina. También, la carta de la representante soviética la describía como una persona con visiones "muy sectarias" (GARF 79284 149, diciembre 1961). Pero, Lebedeva, la representante soviética, consideraba que, a pesar de esta evaluación negativa, la UMA fue en contra de la substitución de Betinelli durante unos años. Entonces, se puede ver que las evaluaciones negativas hechas por las representantes soviéticas y otras integrantes de la cúpula de la FDIM no resultaban en la decisión inmediata. En la práctica, en muchos casos, las decisiones sobre la política de cuadros dirigentes fueron efectuadas por las organizaciones de los países correspondientes (como fue en el caso de dirigentes de partidos comunistas nacionales (Concheiro, Modonesi, y Gutiérrez Crespo, 2007).

Otra parte de la correspondencia, al revés, demuestra que, en algunos casos, las jefas del Comité de las Mujeres Soviéticas en Moscú podían intervenir en la nominación de candidatas a los puestos dirigentes y a participantes de congresos con ponencias claves. Por ejemplo, en 1972, la representante soviética en el Secretariado, Zuhra Rahimbabaeva, recibió carta de Moscú de Xenia Proskurnikova, ${ }^{12}$ que comunicaba que, de acuerdo al Comité Central del Partido Comunista venezolano, el conflicto interno en el partido acabó con su división interna. Por eso, Proskurnikova insistió en que la representante soviética tenía que hacer todo lo posible para prevenir que la dirigente femenina que fue expulsada del partido, Argelia Laya,participara en el consejo de la FDIM (GARF 3 2941, p. 6, 18.01.1972).

Se puede decir que los documentos del Archivo de Moscú demuestran la complejidad de las relaciones de poder dentro de la organización y se descartan las teorías simplificadas del "largo brazo de Moscú". Las dirigentes soviéticas seguramente pensaban en la FDIM como "su" organización, pero debían tener cuidado con las ideas y opiniones de las mujeres de otros países, principalmente, las del "Tercer mundo". Así que Moscú no podía "mandar" y manejar la organización directamente. Y, es por esto que, para Moscú, fue imprescindible seleccionar a las participantes de la dirigencia y de las reuniones principales con la ayuda de la representante soviética en el Secretariado en Berlín.

$\mathrm{Al}$ analizar las relaciones con Moscú, es importante llevar la atención a dos destacadas dirigentes de la FDIM de procedencia argentina Fanny Edelman y Rosa Jasovich.Fanny Edelman, posiblemente la más conocida dirigente argentina en la FDIM, recibió atención de los historiadores del movimiento femenino (Pieper Mooney, 2013b) y dejó su versión sobre la FDIM en sus memorias (1996). En su función dentro de la organización Unión de Mujeres de la Argentina (UMA), Edelman participó en muchas actividades de la FDIM, inclusive en el Primer Congreso de la Federación que tuvo lugar en Asia (Pekín, 1949). Pero su papel más importante en la FDIM corresponde a los años 1970, cuando fue elegida como Secretaria General (1972-1978). En este periodo, trabajaba junto con la Presidenta de la FDIM, la comunista australiana, Freda Brown. 
Los documentos del archivo en Moscú demuestran que ambas dirigentes, Brown y Edelman, tuvieron buen trato con el Comité de Mujeres Soviéticas. Por ejemplo, Edelman discutía su ponencia con la representante soviética en el Secretariado en Berlín, Titova (carta a Proskurnikova de 19.05.1975 - GARF 3 3693, p. 7). En respuesta a esta carta, se puede leer que respecto de la invitación de las mujeres americanas de la FDIM, el Secretariado decidió "que Fanny y Frida viajen vía Moscú. El viaje será efectuado con el vuelo soviético regular y el comité de mujeres Soviéticas les comprará los pasajes de ida y vuelta” (GARF 3 3693, p. 9). En la misma carta, Titova describe también cómo Edelman escribía su presentación para el futuro consejo de la FDIM en Bucarest con la ayuda de Titova (GARF 3 3693, p. 7).

El rol de Fanny Edelman en la FDIM fue especialmente importante durante 1975, declarado por la ONU como el año internacional de las mujeres. Pero Edelman, comunista y voluntaria del Socorro Rojo en España, en 1936, ya había empezado a colaborar con la FDIM prácticamente desde el momento de su fundación, en 1945 (Edelman, 1996). En sus memorias, Edelman describe sus viajes a Moscú en diferentes períodos y sus buenas relaciones con las mujeres del Comité Soviético (Edelman, 1996). El archivo de Moscú contiene más de 30 copias de la carta impresa en la tipografía que fue difundida entre las mujeres argentinas como parte de la campaña de reconocimiento de los éxitos de la URSS y la celebración de aniversario de revolución de octubre en 1950. La carta está dirigida “A las mujeres soviéticas” y, entre otras cosas, dice:

las mujeres argentinas agrupadas en la UMA sabremos hacer honor a vuestro ejemplo, poniendo cada vez más abnegación, más coraje, más emoción en nuestra lucha común, que tan brillantemente vosotras encabezáis (GARF 2 709, p. 9).

Edelman parece ser una de las mujeres latinoamericanas relacionadas con la FDIM que fue conocida y respetada en la Unión Soviética durante los años 70, cuando ejerció como Secretaria General de la FDIM. ${ }^{13}$

A diferencia de Edelman, la otra destacada dirigente de la FDIM de Argentina, Rosa Jasovich Pantaleón, Secretaria General de FDIM entre 1963 y 1967, parece ser menos conocida en la Unión Soviética. Jasovich, hija de inmigrantes judíos en Argentina (como Edelman), fue la Secretaria General durante un período difícil en el que la FDIM tenía que adaptarse al desarrollo de nuevos movimientos femeninos, tanto en el "Tercer Mundo" como en la Europa Occidental. Entonces, Jasovich, representante del PC argentino como Edelman, se hizo primera Secretaria General de la FDIM en representación de los países del "Tercer mundo"; y su posición contribuyó a dar más visibilidad al activismo de las mujeres en América Latina (ver materiales de consejo en Salzburg, 1965 - GARF 3 1396, p. 31). A pesar de las diferencias entre organizaciones de mujeres de diferentes partes del mundo, la cúpula de la FDIM tenía que defender a la Federación como una organización común para todas las mujeres. Esto fue especialmente importante porque la FDIM trataba de demostrar su carácter independiente y lavar su imagen de las acusaciones de "comunismo". Jasovich, por ejemplo, fue responsable de los intentos de la FDIM de ampliar su colaboración con otras organizaciones femeninas transnacionales, inclusive International Council of Women (ICW). ${ }^{14}$

En el mismo tiempo, los documentos internos -la correspondencia entre la representante Soviética en el Secretariado y las dirigentes del Comité de las Mujeres Soviéticas en Moscú- indican que Jasovich tenía menos confianza en Moscú, entre otras cosas, porque estaba dispuesta a meditar sobre la línea ideológica de la Federación. La representante soviética, Zoia Lebedeva, escribió, en 1963, que:

Rosa Jasovich está muy emocionada y preocupada. Ella no puede encontrar la respuesta para las palabras de Fidel en el Congreso de Mujeres Americanas donde él acusó a los líderes de los partidos progresistas de que ellos no pueden encabezar la revolución. Ella está dispuesta a hablar de esto con frecuencia. También, ella escuchó (pero no dice de quién) que el antisemitismo en Europa está creciendo. Todo esto hace que ella "esté menos segura en todo lo que hacemos [en la FDIM y sus dirigentes]" (GARF 4 169, p. 16).

Con base en estos documentos, se puede decir que las representantes de América Latina que pertenecían a la cúpula de la FDIM tenían que actuar, en parte, teniendo en cuenta la opinión de Moscú. No obstante, esto no significa que seguían directamente las órdenes de los países "socialistas". Hemos evidenciado, además, que en la mayoría de los casos se encontraban en la situación de debatirse entre afiliaciones que podían entrar 
en conflicto como integrantes del partido y de la organización de mujeres de su país, como representantes de las mujeres de su país y del "tercer mundo", siendo de América Latina y hablando en nombre de "mujeres de mundo entero".

\section{LA FDIM Y LOS REGIONALISMOS EN LOS AÑOS 1970}

Los años 1970 corresponden al periodo más exitoso en la historia de la Federación. En los 1970, la FDIM mantuvo su estatus de organización transnacional no gubernamental en la ONU, tenía más de 100 paísesmiembros y gozaba de prestigio y visibilidad internacional. La FDIM participó como una organización nogubernamental en los congresos mundiales organizados de la ONU (en México, 1975; Copenhague, 1980, Nairobi, 1985).

El crecimiento de la Federación la convirtió en una de las organizaciones de mujeres más grandes del mundo. En su momento, este crecimiento obligó a su cúpula a pensar en la necesidad de una mayor concentración del trabajo en las regiones para abordar sus problemas específicos. Este proceso parece reflejar los procesos de regionalización característicos del movimiento comunista (Concheiro, Modonesi y Gutiérrez Crespo, 2007). Alrededor del año 1978, la dirigencia de la FDIM tomó la decisión de apertura de dos centros regionales: en Mongolia - para los países asiáticos-y en Cuba-para los latinoamericanos. Pero, como demuestran los documentos del archivo, la apertura del centro en Mongolia encontró muchos problemas. La organización de mujeres de Mongolia demostró poco entusiasmo: podía organizar los cursos solamente por tres meses al año (durante el periodo de las vacaciones universitarios, los estudiantes de los cursos podrían vivir en la residencia estudiantil). Además, el proyecto parece encontrar también muchos escollos de finanzas (GARF 3 4949, p. 1-2). ${ }^{15}$ A diferencia de Mongolia, el centro creado en Cuba de acuerdo a los documentos del archivo fue un éxito. El reporte creado sobre la base de la información de la embajada de la URSS en Cuba y la revista cubana "Mujeres", de abril de 1980, comunicaba que el centro en La Habana había sido abierto el 16 de enero de 1978, en un ambiente muy solemne: la apertura contaba con la presencia de los miembros del gobierno cubano. El centro trabajaba con la ayuda de los planes elaborados por la Federación de Mujeres Cubanas junto con la FDIM y recibía 30 mujeres de diferentes países de América Latina (dos mujeres de cada país), cada tres meses (GARF 3 5799, p. 1).

La descripción corta del programa de educación permite entender que las estudiantes de cursos tenían que aprender historia del movimiento femenino y argumentar sobre derechos de las mujeres. También, las estudiantes de los cursos atendían las clases tanto sobre el uso de los periódicos y de otros medios de comunicación, como sobre el desarrollo económico. Las graduadas de los cursos debían poseer conocimientos sobre los problemas del desarrollo de los países del "Tercer mundo" y, por eso, problemas de racismo, apartheid, subdesarrollo e independencia nacional ocupaban el lugar importante en el programa.

Se puede suponer que, como en el caso de la Unión Soviética, Cuba esperaba ampliar su esfera de influencia con la ayuda del grupo nuevo -las activistas latinoamericanas- que podían demostrar su apoyo al proyecto cubano después de visitar el país. El reporte demuestra, por ejemplo, que todas las estudiantes del curso recibían acceso a la asistencia médica gratuita y tenían posibilidad de visitar diferentes partes del país (GARF 35799 , p. 3).

Repitiendo la experiencia del Comité de Mujeres Soviéticas que acumulaba información sobre el desarrollo del movimiento de mujeres en diferentes países del mundo, ${ }^{16}$ el centro de preparación de cuadros femeninos en la Habana también creó un centro de documentación para acumular información sobre el movimiento femenino en el nivel regional, en América Latina y el Caribe (GARF 3 5799, p. 1). 


\section{Consideraciones Finales}

Los documentos analizados demuestran que el papel de América Latina en la FDIM no siempre fue el mismo; este rol creció con el tiempo. Esto se refiere tanto a la presentación de problemas de las mujeres de la región en las publicaciones oficiales de la Federación, como a la representación de mujeres latinoamericanas en los órganos dirigentes de la FDIM. Los documentos de archivo demuestran, también, que la participación de las mujeres latinoamericanas en la FDIM y su influencia dependía de muchos factores, inclusive de la dinámica de la Guerra Fría, de sus orientaciones políticas y, también, no menos importante, era su posición respecto a la Unión Soviética y su política exterior.

Los documentos demuestran que el ejemplo soviético de la solución de los problemas de las mujeres era central para las mujeres de diferentes países que trabajaban en la organización, inclusive para muchas latinoamericanas. Pero la correspondencia entre las representantes del Comité de mujeres Soviéticas indica que, en algunos casos, las activistas latinoamericanas no estuvieron de acuerdo con la composición de los órganos dirigentes de la federación o con sus decisiones. Otras representantes hacían preguntas difíciles para la cúpula pro-soviética y exigían cambios en diferentes aspectos del trabajo de organización.

Durante el periodo más favorable para el desarrollo de la federación, en los años 1970, que se caracterizaron por el crecimiento de miembros de la federación y de su prestigio internacional, la diversificación interna de la organización creció y significaba al mismo tiempo que, a veces, las mujeres de diferentes partes del mundo preferían concentrarse en los problemas más específicos para la región correspondiente.

Entonces, el periodo más exitoso en la historia de la FDIM coincidió con el crecimiento de regionalismos y formación de diferentes agendas regionales. Este fue el momento oportuno para el crecimiento de la influencia cubana en la Federación, cuando el centro de formación de cuadros femeninos, abierto en la Habana por la FDIM en 1978, jugó un rol importante en este proceso.

Pero la investigación de la actividad de la FDIM en América Latina presenta todavía muchas preguntas que tienen que ser respondidas. Por ejemplo, ¿qué relación hubo entre la política de derechos humanos del PC en cada país y las intervenciones de sus dirigentes en la FDIM en el plano internacional? ¿Qué influencia tenía Cuba en las actividades de la FDIM en el último periodo de la Guerra Fría? En ese sentido, también, es importante investigar las semejanzas y las diferencias de actividades entre las organizaciones de la FDIM en cada país en los años 1960-1980, uno de los períodos menos estudiados aún en relación con esa cuestión. Este artículo intenta contribuir con esa agenda de investigación.

\section{FuenTES}

Archivo Nacional de la Federación Rusa - GARF. Fondo 7928 - El Comité de las Mujeres Soviéticas. Women of the Whole World (WWW)-1969-1980

Zhenschinymira (ZM) - 1953, 1958-1980

\section{Agradecimientos}

Quiero expresar mi gratitud profunda a la Dra. Adriana Valobra por sus comentarios críticos $y$, especialmente, por su generosa ayuda con la corrección del texto.

\section{ReFERENCIAS}

Armstrong, E. (2016). Before Bandung: The Anti-imperialist Women's Movement in Asia and the Women's International Democratic Federation. Signs, 41(2), 305-331. 
Autio-Sarasmo, S. \& K. Miklossy (eds.) (2011). Reassessing Cold War Europe. London: Routledge.

Chase, M. (2015). Revolution Within the Revolution. Women and Gender Politics in Cuba, 1952-1962. Chapel Hill: The University of North Carolina Press.

Concheiro, E., Modonesi, M. y Gutiérrez Crespo, H. (2007). El comunismo: otras miradas desde América Latina. México: UNAM.

de Haan, F. (2012). The Women's International Democratic Federation (WIDF): History, Main Agenda and Contributions (1945-1991). En Th. Dublin and K. Sklar (eds.). Women and Social Movements (WASI) Online Archive, Recuperado de http://alexanderstreet.com/products/women-and-social-movements-international

De Haan, F. (2017). La Federación Democrática Internacional de Mujeres (FDIM) y America Latina, de 1945 a los años setenta. En A. M. Valobra \& M. Yusta (eds.). Queridas Camaradas. Historias iberoamericanas de mujeres comunistas (pp.17-44). Buenos Aires: Miño y Davila.

De Haan, F. (2018). The Global Left-Feminist 1960s. From Copenhagen to Moscow and New York. En Ch. Jian, M. Klimke, M. Kirasirova, M. Nolan, M. Young and J. Waley-Cohen (Eds.), The Routledge Handbook of the Global Sixties (pp. 230-242). London: Routledge.

Donert, C. (2014). Whose Utopia? Gender, Ideology and Human Rights at the 1975 World Congress in East Berlin. En J. Eckel \& S. Moyn The Breakthrough: Human Rights in the 1970s (pp.68-87). Philadelphia: University of Pennsylvania Press.

Edelman, F. (1996). Banderas, Pasiones, Camaradas. Buenos Aires: Dirple.

Gradskova, Y. (2014). The Soviet Union: 'Chile is in Our Hearts.' Practices of Solidarity between Propaganda, Curiosity and Subversion. En K. Christiaens, I. Goddeeris, M. Rodríguez (Eds.), European Solidarity with Chile (pp. 329-346) Frankfurt am Main: Peter Lang.

Gradskova, Y. (2019). Women's International Democratic Federation, the 'Third World' and the Global Cold War from the late-1950s to the mid-1960s. Women's History Review, 29(2), 270-288.

Hajimu, M. (2015). Cold War Crucible. Cambridge Ma.: Harvard University Press.

Ilic, M. (2011). Soviet Women, Cultural Exchange and the Women's international Democratic Federation. En S. Autio-Sarasmo, \& K. Miklossy (eds.), Reassessing Cold War Europe (pp.157-174). London: Routledge.

Kanet, R. (1988). Soviet Propaganda and the process of national liberation. In R. Kanet Soviet Union, Eastern Europe and the Third World (pp.84-114). Cambridge: Cambridge University Press.

McGregor, K. (2013). The Cold War, Indonesian Women and the Global Anti-Imperialist Movement, 1946-1965. En J. Pieper-Mooney \& F. Lanza (Eds.). De-centering Cold War History. Local and Global Change (pp.31-51). London \& New York: Routledge.

McGregor, K. (2016). Opposing Colonialism: the Women's International Democratic Federation and decolonisation struggles in Vietnam and Algeria 1945-1965. Women's History Review, 25(6), 925-944.

Molyneux, M. \& N. Craske (2002). Gender and the Politics of Rights and Democracy in Latin America. Basingstoke: Palgrave.

Pieper Mooney, J. (2013a). Fighting Fascism and Forging New Political Activism: The Women's International Democratic Federation in the Cold War. En J. Pieper-Mooney \& F. Lanza (Eds.). De-centering Cold War History (pp. 52-73) London: Routledge.

Pieper Mooney, J. (2013b). El antifascismo como fuerza movilizadora: Fanny Edelman y la Federación Democrática Internacional de las Mujeres (FDIM). Anuario IEHS, 28.

Stoler, A. L. (2009). Along the Archival Grain: Epistemic Anxieties and Colonial Common Sense. Princeton: Princeton University Press.

Tonchu, E. (2004). Rossiia - zhenskaiasudba. St-Petersburg: Tonchu.

Valobra, A. (2014). "Mujeres-sombra" y "Barbudas": Género y política en el Primer Congreso Latinoamericano de Mujeres, Chile- 1959. Anuario del Instituto de Historia Argentina, 14. En Memoria Académica. Recuperado de: http://www.memoria.fahce.unlp.edu.ar/art_revistas/pr.6729/pr.6729.pdf 
Valobra, A. M. \& M. Yusta (eds.) (2017). Queridas Camaradas. Historias iberoamericanas de mujeres comunistas. Buenos Aires: Miño y Dávila.

Westad, O.A. (2005). The Global Cold War. Third World Interventions and the Making of our Time. Cambridge: Cambridge University Press.

\section{Notas}

1 GARF es el archivo abierto para investigadores/as del exterior.

2 Esta información está basada en la conversación personal con Francisca de Haan, la profesora de Central European University, Budapest, que está estudiando la historia de la FDIM desde hace más de 10 años.

3 Casi ninguno de los ex-países socialistas en el territorio de Europa Oriental o la URSS participa en la actividad de la FDIM después de 1991.

4 Radio Habana Cuba. Mujeres cubanas participaban en la reunión anual del Comité de la FDIM en Namibia, 10.08.2019, http://www.radiohc.cu/noticias/nacionales/198681-mujeres-cubanas-participan-en-reunion-anual-del-c omite-de-la-fdim-en-namibia. La sede de la FDIM se trasladó a Brasil, en 2007, y, ahora, de acuerdo con la información que se puede encontrar en Internet, una mujer salvadoreña es la presidenta de la organización, Lorena Peña. La página de la Federación Democrática Internacional de Mujeres es https://www.fdim.org.sv/

5 Tuve acceso a dos números de 1953 y a la colección más completa de los años 1958-1963, en ruso. Durante los años 1958-1963, la revista pasó a publicarse mensualmente. Para este breve análisis, elegí cuatro números de cada año.

6 Elisa Branco fue una comunista, representante de la Federación de Mujeres de Brasil.

7 La FDIM perdió este status en 1954 como resultado de su apoyo de la posición soviética respecto de la Guerra en Corea (Donert, 2016).

8 Más sobre Popova -la funcionaria de alto nivel de la Unión Soviética- en Ilic, 2011.

9 La necesidad del uso frecuente del concepto "anti-imperialismo" fue especialmente promovido por las participantes de la organización de las mujeres de China Popular.

10 Como escribí antes, la mayoría de las reuniones del Consejo y de la Mesa de la FDIM tenía lugar en Europa. Pero, en pocos casos, la Mesa fue organizada en uno de los países del "Tercer Mundo" -Jakarta (1960), Bamako (1962).

11 Es importante no olvidar también que, después del derrumbe de la URSS y el bloque del Este, la presencia de los países latinoamericanos en la dirigencia de la organización se hizo mucho más fuerte.

12 Proskurnikova era vicepresidenta de la organización soviética. La presidenta, primera astronauta femenina, Valentina Tereshkova, tenía funciones representativas. Proskurnikova, a diferencia de Tereshkova, hizo una carrera muy larga en el partido comunista de la URSS y en el comité de las mujeres soviéticas, empezando el trabajo en NKVD (policía secreta Soviética) en los años 1930 (Tonchu, 2004, p. 361).

13 Edelman tenía 62 años cuando fue elegida Secretaria General de la FDIM, es decir, era una militante con muchos años de experiencia. Cfr. Carta a Fanny del club infantil de amistad, 1976 (GARF, 3 3962, p. 138). Era conocida, también, por acción en la solidaridad internacional (Gradskova, 2014).

14 Es posible que ICW se haya interesado en esos contactos teniendo en cuenta los cambios más profundos en la actitud de la FDIM en la arena internacional: en 1967, la FDIM pudo recuperar su estatus consultivo "B" en ONU -que había perdido después de la Guerra de Corea y las acusaciones de comunismo, en 1954.

15 De otros documentos se puede entender que el centro para preparación de cuadros femeninos para las mujeres de Asia en Ulan-Bator nunca funcionó (GARF 3 5077, p. 97).

16 Gracias a esta decisión soviética, tengo ahora el material para este artículo. 